\title{
Vancomycin intermediate Staphylococcus aureus isolated from a tertiary care hospital in Kolkata
}

\author{
Dr.Susmita Bhattacharya ${ }^{1}$, Dr.Kuhu Pal ${ }^{2}$, Dr.Mitali Chatterjee ${ }^{3}$, \\ Dr.Mandira Banerjee ${ }^{1}$, Dr.Pratip Kumar Kundu ${ }^{4}$, Dr.Swapan Kumar Niyogi ${ }^{5}$ \\ ${ }^{1}$ Department of Microbiology, NRS Medical College, Kolkata, India) \\ ${ }^{2}$ Department of Microbiology, College of Medicine and JNM Hospital, Kalyani, India) \\ ${ }^{3}$ (Department of Microbiology R.G. Kar Medical College, Kolkata, India) \\ ${ }^{4}$ (Department of Microbiology, School of Tropical Medicine, Kolkata, India) \\ ${ }^{5}$ (Department of Microbiology, National Institute of Cholera \& Enteric Diseases, Kolkata, India)
}

\begin{abstract}
With the increasing incidence of methicillin resistant Staphylococcus aureus(MRSA), vancomycin intermediate Staphylococcus aureus (VISA) \& Vancomycin resistant Staphylococcus aureus (VRSA) strains are not a rare phenomenon nowadays. The study was conducted to find out the magnitude of vancomycin resistance and antibiotic susceptibility pattern of those isolates in a tertiary care teaching hospital,Kolkata between August 2009 to July 2011. In this cross sectional study, 714 Staphylococcus aureus were isolated and identified conventionally from various clinical specimens collected from different departments of the hospital. Subsequently, the antimicrobial susceptibility test was performed by Kirby Bauer disc diffusion method as per Clinical and Laboratory Standards Institute (CLSI) guidelines. All the strains found resistant to vancomycin by disc diffusion method were again recruited to E test for confirmation of resistance. Minimum Inhibitory concentration (MIC) of 4 to $8 \mathrm{mg} / \mathrm{L}$ and MIC of $>16 \mathrm{mg} / \mathrm{L}$ were considered as VISA and VRSA respectively. Vancomycin resistance was seen in 21 isolates of $S$. aureus by disc diffusion method. Among them 4 isolates were found to be VISA when confirmed by E test with MIC varying between 4 to $6 \mathrm{mg} / \mathrm{L}$. No VRSA was detected. VISA strains were resistant to almost all the commonly used antibiotics showing sensitivity to linezolid only. Though incidence was not so high but emergence of VISA rang an alarm to the infection control committee of this tertiary care centre of eastern India.
\end{abstract}

Keywords - antibiotic resistance, E test, Eastern India,VISA.

\section{Introduction}

Staphylococcus aureus is one of the most common causes of nosocomial infection like surgical wound infection, blood stream infection, pneumonia etc. The treatment for this bacterium is a problem with the emergence and spread of methicillin resistance gene. Vancomycin is commonly used for treatment of methicillin resistant Staphylococcus aureus (MRSA) ${ }^{1}$. But the incidence of vancomycin-intermediate S. aureus (VISA) and vancomycin-resistant $S$. aureus (VRSA) has been increasing in various parts of the world ${ }^{2}$.

The first reported case of diminished vancomycin susceptibility in a clinical isolate of $S$. aureus was from Japan in $1997^{3}$.It was due to decreased availability of the vancomycin for intracellular target molecules. This was in turn caused by unusually thickened cell wall having dipeptides used for binding vancomycin. This was the first observation of vancomycin-intermediate S. aureus (VISA). Since then, there is a steady increase in the number of cases with VISA and VRSA. This has triggered of alarms in the medical community as S. aureus causes life threatening infections in the hospital as well as in the community.The predicted mechanism of van $A$ gene plasmid-mediated transfer from enterococci to S. aureus was later observed for the first time in 2002 from the United States ${ }^{4}$.This was the first description of VRSA. Recently, Tiwari and Sen ${ }^{5}$ have reported a VRSA which is van A gene negative. Subsequently, VISA and VRSA strains were reported to have been isolated from France ${ }^{4}$ UnitedKingdom ${ }^{6}$, Brazil ${ }^{7}$, Germany $^{8}$ and other countries of the world. As documented reports of VISA/VRSA in India are very few $5,9,10,11,12$. The study was undertaken to monitor the vancomycin susceptibility of MRSA from clinical samples in a tertiary care teaching hospital in Kolkata. Barring two cases - one from Kolkata in $2008{ }^{13}$ and the other from Midnapore in $2011^{14}$, it has not been reported from eastern zone of India to the best of our knowledge. The aim of the present study was to determine the incidence of vancomycin resistance among S. aureus isolates from different clinical specimens and to determine the sensitivity of these isolates to different antimicrobial agents. 


\section{MATERIAL AND METHODS}

A cross sectional study was conducted in a tertiary care teaching hospital of Kolkata, WestBengal, India during the period from August 2009 to July2011.Different clinical specimens like wound swabs, pus, urine, and blood, obtained randomly from inpatients and outpatients of this referral tertiary care hospital were included in the study.

Staphylococci were obtained either as pure culture or as an isolate of a polymicrobial infection. Mannitol salt agar was used as a selective medium for $S$. aureus. These were incubated at $37^{\circ} \mathrm{C}$ for 24 hours. $S$. aureus was identified conventionally based on Gram's stain morphology, colony characteristics, and positive catalase and coagulase tests ${ }^{15}$. The Staphylococcus species were identified on the basis of both slide and tube coagulase test. Slide coagulase was performed for detection of clumping factor present in S. aureus. Tube coagulase test was done for production of free coagulase enzyme. All S. aureus isolates were subjected to susceptibility testing by the Kirby-Bauer disc diffusion method using different antimicrobial agents e.g., penicillin (10 units), chloramphenical (30 $\mu \mathrm{g})$, clindamycin $(2 \mu \mathrm{g})$,cefotaxime $(30 \mu \mathrm{g})$,ciprofloxacin $(5 \mu \mathrm{g})$, cefoxitin $(30 \mu \mathrm{g})$, co-trimoxazole $(25 \mu \mathrm{g})$, erythromycin $(15 \mu \mathrm{g})$, fusidic acid $(10 \mu \mathrm{g})$, gentamicin $(10 \mu \mathrm{g})$, linezolid $(30 \mu \mathrm{g})$, mupirocin $(5 \mu \mathrm{g})$, netilmycin(10 $\mu \mathrm{g})$, ofloxacin $(5 \mu \mathrm{g})$ oxacillin $(1 \mu \mathrm{g})$, piperacillin-tazobactam(100/10), vancomycin $(30 \mu \mathrm{g})(\mathrm{Hi}$-media).

The MRSA strains were identified using oxacillin disc $(1 \mu \mathrm{g})$, cefoxitin disc $(30 \mu \mathrm{g})$ as reported by various authors ${ }^{16}$ and slidex staph latex agglutination tests (bioMerieux) which detects methicilin resistance in Staphylococci based on the production of low-affinity PBP2a, which is encoded by the mec A gene .

Zone diameter of the test strain was measured in millimeter with a template. The isolates were considered vancomycin resistant if the zone of inhibition was $14 \mathrm{~mm}$ or less.

The strains found to be resistant to vancomycin by disc diffusion test were resorted to $\mathrm{E}$ test (bioMerieux) to determine the minimum inhibitory concentration (MIC) and confirmation of their resistance status.

0.5 McFarland equivalent inoculums, prepared by using 18-24 hrs old culture was inoculated on the Mueller Hinton agar plates on which E test strip was implanted according to the user guideline. Plates were incubated at $35^{\circ} \mathrm{C}$ for $24 \mathrm{~h}$ before assessing the visible growth. The MIC is the value at which the elliptical zone of inhibition converges on the strips. Isolates with a vancomycin MIC 4 to $8 \mathrm{mg} / \mathrm{L}$ were identified as vancomycin-intermediate, isolates with a vancomycin MIC $>16 \mathrm{mg} / \mathrm{L}$ were identified as vancomycin-resistant S.aureus(CLSI, 2007) ${ }^{17}$. Quality control was performed using the S. aureus ATCC 29213 strain and ATCC 43300 as MRSA control strain.

Institutional Ethics Committee clearance has been obtained before conducting this study. Statistical analysis: The data collected were put into MS-Excel sheets for analysis. Percentages were used for interpretation of the data in this study.

\section{RESULT}

From the various clinical samples 714 Staphylococcus aureus were isolated during the study period. Among the isolates 280 were MRSA strain (39.1\%). Among 280 MRSA, 21strains were found to be resistant to vancomycin by disc diffusion test. Of these strains, 4 were found to be VISA showing an MIC range between 4-6 mg/L, when tested by E test (bioMerieux). "Fig.1" shows E test finding of VISA 4 strain with MIC of $6 \mathrm{mg} / \mathrm{L}$. The MIC for 276 of 280 isolates $(98.57 \%)$ for vancomycin was $\leq 2 \mathrm{mg} / \mathrm{L}$ indicating vancomycin sensitive strains. None of the isolates were resistant to vancomycin by E test(MIC in the range of 16-64 mg/L). Zone of inhibition of 3 VISA strains were $13 \mathrm{~mm}$ and one was found $14 \mathrm{~mm}$ in disc diffusion method.

All the isolated VISA strains were resistant to penicillin, cefotaxime, co-trimoxazole, cefoxitin, ciprofloxacin, oxacillin, gentamicin, netilmycin, ofloxacin, piperacillin-tazobactam. Linezolid was the most effective drug against VISA showing $100 \%$ sensitivity. Fifty percent of VISA were sensitive to clindamycin and fusidic acid. Details of VISA strains was given in "Table I"

\section{DisCussion}

There is a global epidemic of methicillin resistant Staphylococcus aureus (MRSA) giving rise to increased morbidity and mortality. It is now responsible for around 30\% or more of all serious infections though this figure varies ${ }^{10,18}$. There is an increased chance to cause cross infection and MRSA also has the ability to colonize individuals for months and years. Vancomycin was the only answer for those MRSA, so intensive use of this antibiotic might create a considerable selection pressure for emergence of the resistance to this glycopeptide ${ }^{19}$.

In this present study 39.21\% (280/714) of Staphylococcus aureus were MRSA strains and only $2.09 \%$ (21/714) strains were resistant to vancomycin by disc diffusion testing. But a high rate of vancomycin resistance (79.73\%) was reported in a study in sikkim ${ }^{20}$ but resistance was not substantiated by MIC testing. In our study only $4(0.56 \%)$ strains were found to be VISA when subjected to E test whereas in a study in northern India 6 
$(0.76 \%) \quad$ VISA strains and 2(0.25\%) VRSA strains $\quad$ were reported $^{5}$.

In this study, incidence of VISA cases has been found in both pediatric and adult age group and it involved both the male and female population. Moreover it has been isolated from different wards like NICU, Surgery and Gynecology wards. So, it can be said that VISA is prevalent in different wards of the hospital involving all age group of patients indicating the magnitude of the problem. This wide distribution of VISA strains is to be kept in mind while formulating treatment policy of infections in the hospital.

Samples were collected from indoor as well as from outdoor patients of this hospital. But we have found the VISA cases only in the hospitalized patients, though there are cases reported from the community in other studies $^{21}$.

In the present study, isolated VISA showed resistance to a wide range of antimicrobial agents. Only linezolid stood as effective drug against all those strains.

MIC of VISA ranges between 4 to $6 \mathrm{mg} / \mathrm{L}$ in this study whereas MIC of VISA found in north India ${ }^{5}$ were $8 \mathrm{mg} / \mathrm{L}$ in 4 isolates and $16 \mathrm{mg} / \mathrm{L}$ in 2 isolates and VISA strain in South India ${ }^{10}$ showed MIC of $5 \mathrm{mg} / \mathrm{L}$. One of the causes of difference of the MIC range may be due to the difference in the procedures. In these two studies MIC was determined by agar dilution method and broth dilution method respectively whereas in our study E test kit was employed.

VRSA strain reported in Kolkata ${ }^{13}$ was senisitive to gentamicin and ciprofloxacin but all VISA in our study were resistant to gentamicin and ciprofloxacin and VISA strain isolated in south India ${ }^{10}$ were also resistant to these two drugs. VISA strains were found to be multidrug resistant against a large number of currently available antimicrobial agents, thereby reducing the treatment options for the treating clinicians. In the patients in the paediatric age group, it is found that clindamycin is an alternate, safe and cost effective drug than linezolid in case of VISA $^{22}$ but in our study only $50 \%$ VISA were sensitive to clindamycin.

Though the true mechanism of vancomycin resistance in S. aureus is not known, the problem of antibiotic resistance in developing country like ours seems to be related to irrational antibiotic usage as it is easily available at medicine shops without prescription leading to irregular and irrational treatment; improper dosage and inadequate duration of therapy are other common causes in our country. There is also injudicious use of antibiotics in hospitals; uncontrolled use in agriculture, animal husbandry, fisheries; poor implementation of infection control policies and overcrowding in the hospital set up are resulting in building of selective pressure on vancomycin resistance ${ }^{23}$. Moreover, widespread use of vancomycin to treat infections caused by MRSA and other gram-positive cocci has led to the emergence of vancomycin resistance ${ }^{5}$.

The current study only indicates the tip of the iceberg. We should undertake more such studies in future to fight against the rising menace of antibiotic resistance. More research should be done to find out better treatment policies, effective and cheaper alternative antibiotics in a developing country like ours. The study findings were shared with the hospital infection control committee to help them formulating a new antibiotic policy so that primary caregivers can use antibiotic rationally.

Teicoplanin, another glycopeptides was not tested in this study, so we cannot compare resistance patterns of this drug with that of vancomycin. We have not performed sensitivity against the newer drugs available against S.aureus e.g., Daptomycin,Quinupristin, Tigecycline in this study. These were the short comings of this study.

The VISA strains are preserved in glycerol broth and kept at $\left(-20^{\circ} \mathrm{C}\right)$ to detect the presence or absence of the van $A$ gene in future study.

\section{FIGURES AND TABLES}

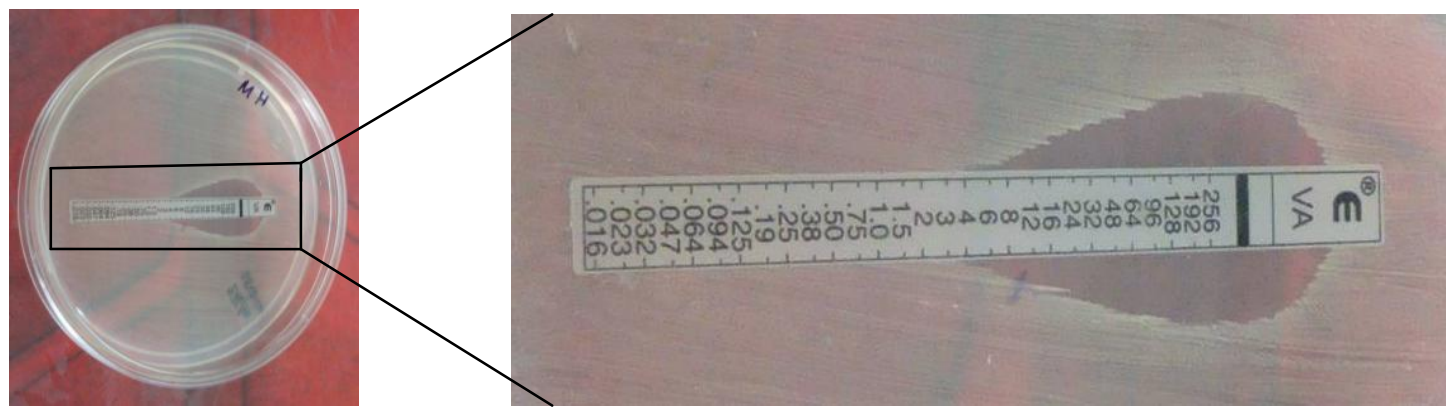

Fig. I E test showing VISA 4 strain (MIC 6mg/L) 
Table I Details of Vancomycin Intermediate Staphylococcus aureus Strains

\begin{tabular}{|c|c|c|c|c|c|c|c|}
\hline Isolate no. & Specimen & Ward & Age & Sex & $\begin{array}{l}\text { MIC of } \\
\text { VA }\end{array}$ & Resistance & Sensitive \\
\hline VISA 1 & $\begin{array}{l}\text { Wound } \\
\text { swab }\end{array}$ & $\begin{array}{l}\text { Obst. } \\
\text { Gynae }\end{array}$ & $30 \mathrm{yrs}$ & $\mathrm{F}$ & $4 \mathrm{mg} / \mathrm{L}$ & $\begin{array}{l}\text { C,CD,CN,CO,CE,CF,E,FC,P, } \\
\text { G,MU,NET,OF,OX,PIT,VA }\end{array}$ & LZ \\
\hline VISA 2 & $\begin{array}{l}\text { Umbilical } \\
\text { swab }\end{array}$ & NICU & 4days & M & $4 \mathrm{mg} / \mathrm{L}$ & $\begin{array}{l}\text { C,CE,CF,CN,CO,E,FC,G,M, } \\
\text { U,NET,OF,OX,P,PIT,VA }\end{array}$ & $\mathrm{LZ}, \mathrm{CD}$ \\
\hline VISA 3 & $\begin{array}{l}\text { Wound } \\
\text { swab }\end{array}$ & Surgery & $42 \mathrm{yrs}$ & M & $5 \mathrm{mg} / \mathrm{L}$ & $\begin{array}{l}\text { C,CD,CE,CF,CN,CO,E,G,P, } \\
\text { MU, NET,OF,OX,PIT,VA }\end{array}$ & LZ, FC \\
\hline VISA 4 & $\begin{array}{l}\text { Wound } \\
\text { swab }\end{array}$ & $\begin{array}{l}\text { Paed. } \\
\text { Surgery }\end{array}$ & $5 \mathrm{yrs}$ & $\mathrm{F}$ & $6 \mathrm{mg} / \mathrm{L}$ & $\begin{array}{l}\text { C,CE,CF,CO,CN,G,NET,P, } \\
\text { OF,OX,PIT,VA }\end{array}$ & $\begin{array}{c}\text { LZ, CD, } \\
\text { E, F, } \\
\text { MU }\end{array}$ \\
\hline
\end{tabular}

$\mathrm{C}=$ Chloramphenicol; $\mathrm{CD}=$ Clindamycin $\mathrm{CE}=$ Cefotaxime; $\mathrm{CF}=$ Ciprofloxacin; $\mathrm{CN}=\mathrm{Cefoxitin}$; $\mathrm{CO}=$ Cotrimoxazole; $\mathrm{E}=$ Erythromycin; $\mathrm{FC}=$ Fusidic acid; $\mathrm{G}=$ Gentamicin; $\mathrm{LZ}=$ Linezolid; MU= Mupirocin; $\mathrm{NET}=$ Netilmycin $; \mathrm{OF}=$ Ofloxacin $; \mathrm{OX}=$ Oxacillin; $\mathrm{P}=$ Penicillin $; \mathrm{PIT}=$ Piperacillin-Tazobactam; $\mathrm{VA}=$ Vancomycin

\section{Conclusion}

For all practical purposes, vancomycin was the only drug available for MRSA till now. The emergence of vancomycin-intermediate staphylococcal isolates in this part of the country is of great concern as hardly any treatment options are left. So, this study emphasizes the need for continuous monitoring of MIC levels of vancomycin in MRSA, and the importance of its rational use in the infection-control practices to prevent transmission of MRSA as well as VISA strains.

\section{ACKNOWLEDGEMENTS}

We are indebted to "The West Bengal University of Health Sciences" in allowing us to publish this paper as a part of the thesis work entitled "Characterization of methicillin resistant Staphylococcus aureus isolated from surgical wound infection in a tertiary care hospital in Kolkata by phenotypic and genotypic methods" (PhD in Medicine) of Dr. Susmita Bhattacharya, Assoc. Prof. Microbiology, NRS Medical College, Kolkata.

\section{REFERENCES}

[1] M. Prakash, V. Karthikeyan, Karuppusamy, S., Karmegam. Journal of Ecotoxicology and Environmental Monitoring2006;16:387389

[2] H.K.Tiwari, D. Sapkota, M. Ranjan Sen. Infection anDrug Resistance 2008;1:57-61.

[3] Hiramatsu K, Hanaki H, Ino T, Oguri YT, Tenover FC. J Antimicrob Chemother 1997; 40:135-36.

[4] Poly MC, Grelaud C, Martin C, de Lumley L, Denis F. First clinical isolate of vancomycin-intermediate Staphylococcus aureus in a French hospital. Lancet 1998; 351, Issue 9110:1212.

[5] Tiwari HK, Sen MR. Emergence of vancomycin resistant Staphylococcus aureus(VRSA) from a tertiary care hospital from northern part of India Infect Dis 2006;6:156.

[6] Veronica N. Kos, Christopher A. Desjardins et al. Comparative Genomics of Vancomycin-Resistant Staphylococcus aureus Strains and Their Positions within the Clade Most Commonly Associated with Methicillin-Resistant S. aureus Hospital-Acquired Infection in the United States May 2012 mBio vol. 3 no. 3 e00112-12

[7] Palazzo ICV, Araujo MLC, Darini ALC: First Report of Vancomycin-Resistant Staphylococci Isolated from Healthy Carriers in Brazil. J Clin Microbiol 2005; 43:179-185

[8] Bierbaum G, Fuchs K, Lenz W, Szekat C, Sahl HG: Presence of Staphylococcus aureus with reduced susceptibility to vancomycin in Germany.Eur J Clin Microbiol Infect Dis 1999;18:691-696

[9] Assadullah S, Kakru DK, Thoker MA, Bhat FA, Hussain N, Shah A.. Emergence of low level vancomycin resistance in MRSA. Indian J Med Microbiol 2003;21: 196-198

[10] G. A. Menezes B. N. Harish S. Sujatha K. Vinothini and S. C. Parija. Emergence of vancomycin intermediate Staphylococcus species in southern India .J Med Microbiol July 2008;57(7): 911-912

[11] Loomba PS, Taneja J, Mishra B. Methicillin and Vancomycin Resistant S. aureus in Hospitalized Patients. J Glob Infect Dis. 2010 Sep;2(3):275-83

[12] Chandra Mohan Reddy.P,Venu Babu Thati, Shivannavar C.T. and Subhash Chandra M. Gaddad. Vancomycin resistance among methicillin resistant Staphylococcus aureus isolates in Rayalaseema region Andhra Pradesh, South India. World Journal of Science and Technology 2012, 2(1):06-08

[13] Biswajit Saha, Anil K. Singh, Abhrajyoti Ghosh, Manjusri Bal. Identification and characterization of a vancomycinresistant Staphylococcus aureus isolated from Kolkata (South Asia). J Med Microbiol January 2008; 57(1): 72-79

[14] S P Chakraborty, S K Mahapatra, M Bal, Somenath Roy.Isolation and Identification of Vancomycin Resistant Staphylococcus aureus from Post Operative Pus Sample. AlAmeen J Med Sci 2011;4 (2 ):152 -168

[15] Koneman Elmer, Winn Washington, Allen Staphen, Procop Gary et al. Koneman's Color Atlas \& Textbook of Diagnostic Microbiology, 6th edition; 2006: 643 - 648.

[16] Amita Jain, Astha Agarwal and Raj Kumar Verma. Cefoxitin disc diffusion test for detection of meticillin-resistant staphylococci, Journal of Medical Microbiology (2008), 57, 957-961

[17] Clinical and Laboratory Standards Institute. Performance standards for antimicrobial susceptibility testing, 17th informational supplement (M100-517). Wayne, Pa: Clinical and Laboratory Standards Institute; 2007. 
[18] Borriello Peter S, Murray R. Patrick, Funke Guido in Topley \& Wilson's Microbiology \& Microbial infections Bacteriology; 2: 793 $-4$

[19] Pray, L. Antibiotic resistance, mutation rates and MRSA. Nature Education.2008; $1: 1$

[20] Tsering DC, Pal R, Kar S. Methicillin-Resistant Staphylococcus Aureus: Prevalence and Current Susceptibility Pattern in Sikkim.Journal of Global Infectious Disease 2011; vol3(1):9-13

[21] Goud R, Gupta S, Neogi U, Agarwal D, Naidu K, Chalannavar R, Subhaschandra G. Community prevalence of methicillin and vancomycin resistant Staphylococcus aureus in and around Bangalore, southern India. Rev Soc Bras Med Trop. 2011 May-Jun; 44(3):309-12.

[22] Catherine Liu, Arnold Bayer, Sara E. Cosgrove, Robert S. Daum, Scott K. Fridkin, Rachel J. Gorwitz et al. Clinical Practice Guidelines by the Infectious Diseases Society of America for the Treatment of Methicillin-Resistant Staphylococcus aureus Infections in Adults and Children: Executive Summary. Clin Infect Dis. 2011; 52 (3): 285-292.

[23] Abhay Dhand and George Sakoulas. Reduced vancomycin susceptibility among clinical Staphylococcus aureusisolates ('the MIC Creep'): implications for therapy. F1000 Med Rep. Feb 2012; 4: 4. 